\title{
共价有机框架在能源存储及转化中的研究进展
}

\author{
彭正康丁慧敏＼cjkstart陈如凡＼cjkstart高超＼cjkstart汪成* \\ (武汉大学分子与科学学院 武汉 430072)
}

\begin{abstract}
摘要 共价有机框架(Covalent Organic Frameworks, COFs)是一类由有机结构单元通过共价键连接形成的多孔框架晶体 材料, 具有密度低、比表面积大和热稳定性高等特点, 在分子吸附与分离、传感、催化、光电器件等领域存在着广阔 的应用前景. 近年来, 基于其固有结构特点, 二维 COFs 在能源领域中的应用潜力也逐渐引起了科学界的关注. 本文主 要综述了二维 COFs 材料在能源存储(锂离子电池、锂硫电池、超级电容器、燃料电池)和能源转化(水分解反应以及 $\mathrm{CO}_{2}$ 还原反应)等方面的研究进展, 并对其研究前景进行了展望.
\end{abstract}

关键词 共价有机框架; 二次电池; 超级电容器; 燃料电池; 水分解 $\mathrm{CO}_{2}$ 还原

\section{Research Progress in Covalent Organic Frameworks for Energy Storage and Conversion}

\author{
Peng, Zhengkang Ding, Huimin Chen, Rufan Gao, Chao Wang, Cheng* \\ (College of Chemistry and Molecular Sciences, Wuhan University, Wuhan 430072)
}

\begin{abstract}
Covalent organic frameworks (COFs) are a class of porous crystalline materials consisting of organic units connected through covalent bonds. Due to their low density, high surface area and high thermal stability, COFs have found interesting applications in many fields, including molecular adsorption and separation, sensing, catalysis and optoelectronics devices. In particular, two-dimensional (2D) COFs have attracted increasing attention in energy fields. In this perspective, the applications of 2D COFs in energy storage (lithium ion batteries, lithium-sulfur batteries, supercapacitor and fuel cells) and energy conversion (water splitting and reduction of carbon dioxide) are reviewed. In addition, we will also discuss the remaining challenging issues.
\end{abstract}

Keywords covalent organic frameworks; rechargeable batteries; supercapacitor; fuel cells; water splitting; $\mathrm{CO}_{2}$ reduction

\section{1 引言}

共价有机框架 (Covalent Organic Frameworks, COFs) 是一类由有机结构单元通过共价键连接形成的晶 态有机多孔材料 ${ }^{[1]}$, 具有比表面积大、结晶性好、孔隙 率高等特点, 受到科研人员的广泛关注. 与其它有机多 孔材料如共轭微孔聚合物 ${ }^{[2]}(\mathrm{CMP})$ 、多孔芳香骨架 ${ }^{[3]}$ $(\mathrm{PAF})$ 、超交联聚合物 ${ }^{[4]}(\mathrm{HCP})$ 等相比, COFs 具有有序的 堆积结构和良好的结晶性 ${ }^{[1 \mathrm{c}]}$. 依据“框架化学”(Reticular Chemistry), 研究人员可对 COFs 材料的骨架和孔道进 行设计, 并对其结构与功能进行更加精确的调控 ${ }^{[1 \mathrm{a}, 1 \mathrm{~d}]}$. 自 2005 年 Yaghi 等 ${ }^{[5]}$ 报道首例 COF 以来, COFs 受到研 究人员的广泛关注. 目前 COFs 已被应用于气体存储与 分离 ${ }^{[6]}$ 、传感 ${ }^{[7]}$ 、催化 ${ }^{[8]}$ 、光电器件 ${ }^{[9]}$ 等诸多领域, 并取 得了一系列重要进展.

目前 COFs 的合成方法主要包括溶剂热法、研磨法、 离子热法、界面合成法和微波法等. 根据拓扑结构, $\mathrm{COFs}$ 可分为二维 COFs 和三维 COFs. 在二维 COFs 中,
有机小分子通过共价键构成片层结构，这种层状堆积的 共轭体系赋予其独特的光电性能. 在三维 COFs 中, 有 机小分子通过共价键连接形成立体三维网状结构, 使其 在气体吸附及分离、催化等方面的应用更具优势.

近年来，基于二维 COFs 的可设计性强、孔隙率高、 结晶性好以及稳定性优异等特点, 其在能源存储和能源 转化领域中展现出了巨大的应用前景 ${ }^{[10]}$. 首先, 由于其 构筑基元广、合成方法多，科研人员可从不同功能基团 出发来构筑功能性(如氧化还原) $\mathrm{COFS}^{[11]}$; 同时，根据实 际应用需求, 可对 COFs 孔道环境进行调控, 以期获得 性能优异的能源存储或能源转化材料 ${ }^{[1 a]}$. 其次, 二维 COFs 的多孔结构不仅为活性物质的负载提供空腔, 为 离子运输提供通道, 而且有利于客体分子与活性位点的 充分接触 ${ }^{[10 \mathrm{f}]}$. 接着, 二维 COFs 高度有序的堆积结构为 载流子传输提供了良好通道, 有利于激发态电子分离和

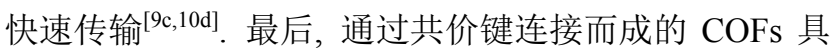
有良好的化学稳定性和热稳定性, 有利于维持能源存储 或能源转化过程中的循环稳定性 ${ }^{[12]}$. 本文主要综述了

\footnotetext{
*E-mail: chengwang@whu.edu.cn

Received April 7, 2019; published May 20, 2019.

Project supported by the National Natural Science Foundation of China (No. 21572170).

项目受国家自然科学基金(No. 21572170)资助.
} 
近年来二维 COFs 在能源存储(锂离子电池、锂硫电池、 超级电容器、燃料电池) 和能源转化(水分解反应以及 $\mathrm{CO}_{2}$ 还原反应)两方面的研究进展, 并对该领域进行了 总结与展望.

\section{2 二维 COFs 在能源存储中的应用}

随着化石能源的不断消耗, 可再生能源的开发与利 用受到越来越多的关注. 能源存储作为能源开发和利用 过程中的重要环节, 对可再生能源的利用和开发具有重 要意义 ${ }^{[13]}$. 二次电池和超级电容器是应用比较广泛的 电储能设备, 其储能性能很大程度上取决于电极材料的 性能. 为开发能量密度大和转化效率高的电储能装置, 目前已有大量新型材料被用于电极材料的研究 ${ }^{[14]}$. 二 维 COFs 作为一种可设计性强的新型有机多孔材料, 可 通过对其骨架中功能基团的调控和孔道结构的设计, 获 得具有较好电储能性能的电极材料. 本部分将从锂离子 电池、锂硫电池、燃料电池和超级电容器四个方面介绍 二维 COFs 在这一领域的研究进展.

\section{1 锂离子电池}

锂离子电池是当前最普遍的商业化储能器件, 被广 泛应用于便携式移动设备中. 锂离子电池的性能主要取 决其正极材料, 传统的正极材料通常为含锂的过渡金属 氧化物或磷酸盐, 然而这些无机材料理论容量低 ${ }^{[15]}$, 生 产过程中易产生环境污染, 且在过充过程中会放热, 存 在安全隐患 ${ }^{[16]}$. 有机正极材料因其成本低、易合成、环 境友好且氧化还原性质可调控, 吸引了研究者们的兴 趣 ${ }^{[17]}$. 目前研究较多的有机正极材料有导电聚合物 ${ }^{[18]}$ 、

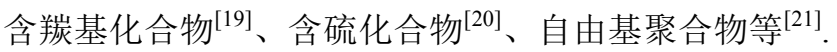
然而这类材料存在比容量低、离子迁移率低, 体积变化 大，活性物质易溶解流失的问题，限制了其进一步发 展 ${ }^{[22]}$. COFs 作为一种稳定的有机多孔材料, 在使用过程 中能够保持其结构的完整, 可避免有机活性物质的溶 解, 有助于提高正极材料的循环稳定性. Jiang 课题组 ${ }^{[23]}$ 2015 年以菜四碳二酰亚胺为前体合成了 DTP-ANDI$\mathrm{COF}$, 并首次研究其在锂离子电池正极中的应用. 在 $200 \mathrm{~mA} \cdot \mathrm{g}^{-1}$ 电流密度下, 该材料中初始活性单元利用率 仅为 $48 \%$. 为提高 DTP-ANDI-COFs 活性单元利用率, 该课题组将 DTP-ANDI-COF 生长于碳纳米管(Carbon Nanotubes, CNTs)表面, 构筑了基于 COF@CNTs 复合材 料的锂离子电池. 结果表明, DTP-ANDI-COF@CNTs 在 $200 \mathrm{~mA} \cdot \mathrm{g}^{-1}$ 电流密度下, 其活性单元的利用率提升至 $90 \%$, 在 $1000 \mathrm{~mA} \cdot \mathrm{g}^{-1}$ 的高电流密度下仍能维持在 $71 \%$. 2017 年, Wang 课题组 ${ }^{[24]}$ 利用球磨的方法将 DAAQ-TFPCOF 剥离得到薄层的 DAAQ-ECOF(如图 1 所示), 研究 结果表明在 $500 \mathrm{~mA} \cdot \mathrm{g}^{-1}$ 电流密度下 DAAQ-ECOF 比未 剥离的 DAAQ-TFP-COF 具有更高的稳定比容量, 且经 过 1800 次循环测试, DAAQ-ECOF 的比容量仍为其理论 容量的 $98 \%$. 且 DAAQ-ECOF 表现出的 $\mathrm{Li}^{+}$扩散系数为
$6.94 \times 10^{-11} \mathrm{~cm}^{2} \cdot \mathrm{s}^{-1}$, 要高于常见的商用正极材料.

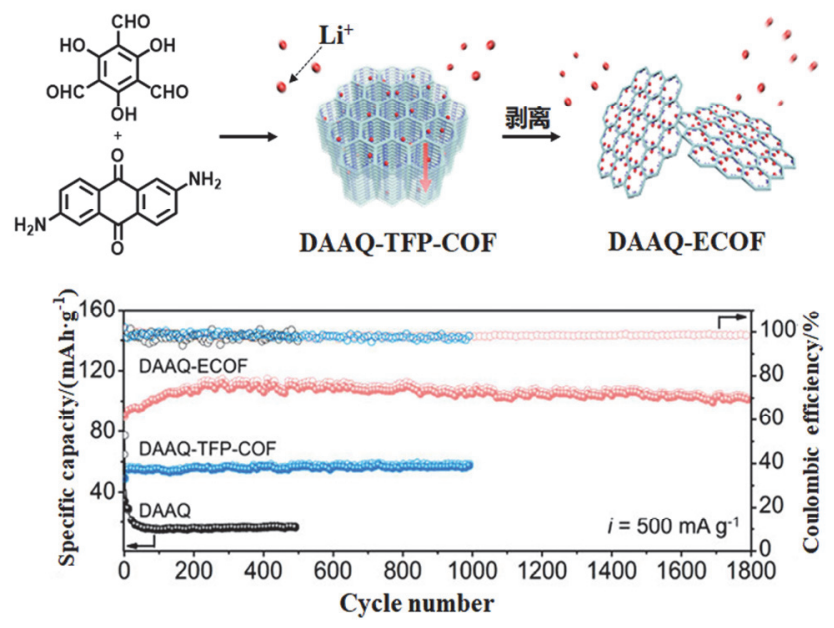

图 1 DAAQ-ECOF 的合成以及其在 $500 \mathrm{~mA} \cdot \mathrm{g}^{-1}$ 电流密度下的循环性 能

Figure 1 Synthesis of DAAQ-ECOF and its cyclic performance at 500 $\mathrm{mA} \cdot \mathrm{g}^{-1}$

COFs 除了可用于构筑正极材料, 还可用于锂离子 电池负极材料. 2018 年 Wang 课题组 ${ }^{[25]}$ 以均三苯甲醛和 对苯二胺为前体合成的 $\mathrm{COF}$ 生长到碳纳米管表面, 并 将得到的 COF@CNTs 用于构筑锂离子电池. 在 100 $\mathrm{mA} \cdot \mathrm{g}^{-1}$ 的电流密度下经过 500 次循环, 其稳定比容量仍 高达 $1021 \mathrm{mAh} \cdot \mathrm{g}^{-1}$ (如图 2 所示). 复合材料中 COF 所贡 献的比容量为 $1536 \mathrm{mAh} \cdot \mathrm{g}^{-1}$, 要明显高于常见的金属氧 化物 $\left(\mathrm{CuO} 、 \mathrm{Fe}_{2} \mathrm{O}_{3} 、 \mathrm{Co}_{3} \mathrm{O}_{4} 、 \mathrm{NiO}\right.$ 等)负极的容量. 理论计 算表明, $\mathrm{COFs}$ 框架中的 $\mathrm{C}=\mathrm{N}$ 键及苯环均可存储 $\mathrm{Li}^{+}$, 极大提升了COF@CNTs 复合材料的理论容量.

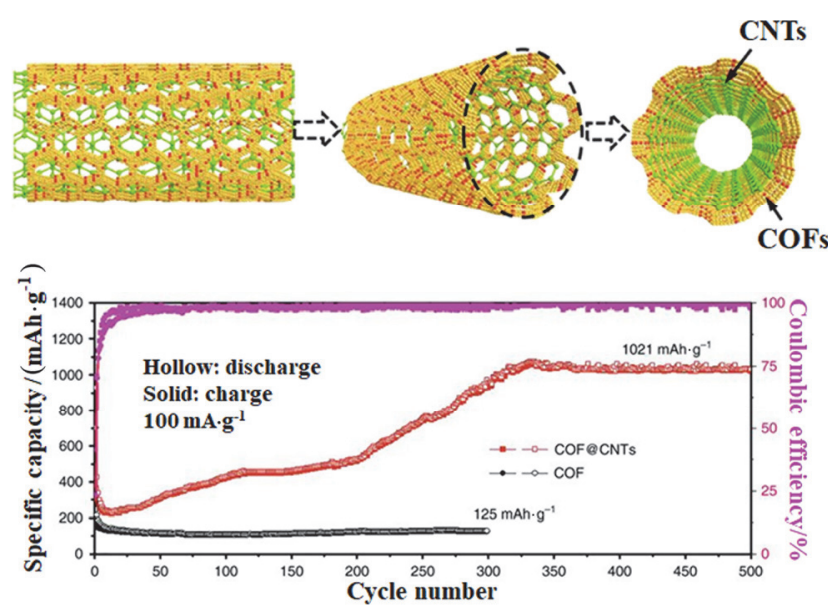

图 $2 \mathrm{COF} @ \mathrm{CNT}$ 的构造及其 $100 \mathrm{~mA} \cdot \mathrm{g}^{-1}$ 电流密度下循环性能 Figure 2 Construction of COF@CNT and its cyclic performance at 100 $\mathrm{mA} \cdot \mathrm{g}^{-1}$

固态 $\mathrm{Li}^{+}$传导材料是构筑固态锂电池的关键. 固态 $\mathrm{Li}^{+}$传导材料有无机氧化物、硫化物 ${ }^{[26]}$ 如氧化物陶瓷、 硫化玻璃陶瓷，这些材料具有良好的离子导电性，但电 
化学稳定性低、晶界电阻高、机械灵活性差 ${ }^{[27]}$. 与无机 $\mathrm{Li}^{+}$传导材料相比, 固态聚合物电解质电化学稳定性好、 $\mathrm{Li}^{+}$离子迁移数高, 可用于固态 $\mathrm{Li}^{+}$传导, 但其离子导电 率低 ${ }^{[27 c, 28]}$. COFs 具有良好的晶形结构且具有稳定的有 机骨架和开放孔道结构, 为 $\mathrm{Li}^{+}$传导提供了通道, 可用 于制备固态 $\mathrm{Li}^{+}$传导材料, 对构筑高能量、高功率的固 态锂电池具有重要的价值. 2016 年, Zhang 课题组 ${ }^{[29]}$ 利 用螺喼酸酯构筑了离子型 ICOF-1 和 ICOF-2, 并将 ICOF-2 首次用于制备固态 $\mathrm{Li}^{+}$传导材料. 研究表明, ICOF-2 室温下表现出 $\mathrm{Li}^{+}$导电率为 $3.05 \times 10^{-5} \mathrm{~S} \cdot \mathrm{cm}^{-1}$. 2018 年 Chen 课题组 ${ }^{[30]}$ 制备了阳离子骨架 CON-TFSI, 可有效屏蔽库仑作用, 抑制离子对的形成, 在 $70{ }^{\circ} \mathrm{C}$ 下 对 $\mathrm{Li}^{+}$的电导率达到 $2.09 \times 10^{-4} \mathrm{~S} \cdot \mathrm{cm}^{-1}$. 随后, Wang 课 题组 ${ }^{[31}$ 将聚乙二醇(PEG) 掺入 COFs 孔道中获得了一系 列装载 PEG 的 COFs, 利用 PEG 对 $\mathrm{Li}^{+}$的良好传输特性 和 COFs 骨架特点, 所制备的 PEG-Li ${ }^{+} @ \mathrm{~EB}-\mathrm{COF}-\mathrm{ClO}_{4}$ 在 $120{ }^{\circ} \mathrm{C}$ 下对 $\mathrm{Li}^{+}$的电导率达到 $1.78 \times 10^{-3} \mathrm{~S} \cdot \mathrm{cm}^{-1}$. 与 其他晶型有机多孔材料相比, PEG-Li ${ }^{+} @ \mathrm{~EB}-\mathrm{COF}-\mathrm{ClO}_{4}$ 表现出了更加优异的 $\mathrm{Li}^{+}$传导特性. 不同于 COFs 装载 PEG 分子的策略, Jiang 课题组 ${ }^{[32]}$ 通过直接构筑法将聚 氧化乙烯(PEO)连接至 $\mathrm{COF}$ 骨架中, 并通过 $\mathrm{Li}^{+}$配位制 备含 $\mathrm{Li}^{+}$的 $\mathrm{Li}^{+} @$ TPB-DMTP-COF 和 $\mathrm{Li}^{+} @$ TPB-BMTPCOF. 研究表明, 相比于骨架中不含 PEO 的 COF, $\mathrm{Li}^{+} @$ TPB-BMTP-COF 的 $\mathrm{Li}^{+}$导电率提升了三个数量级. 此外, Horike 课题组 ${ }^{[33]}$ 通过直接构筑法将不同长度的 PEO 键 连至 COF 骨架中, 并构筑了不同 PEO 密度 COF-PEO- $x$ ( $x$ 为 PEO 单元数量), 并首次将其用于全固态锂电池. 结果表明, COF 材料的 $\mathrm{Li}^{+}$导电性受 PEO 单元密度的影 响, $200{ }^{\circ} \mathrm{C}$ 下 COF-PEO-9-Li 的 $\mathrm{Li}^{+}$导电率为 $1.33 \times 10^{-3}$ $\mathrm{S} \cdot \mathrm{cm}^{-1}$.

\section{2 锂硫电池}

相对于锂离子电池, 锂硫电池具有高理论容量 $\left(1672 \mathrm{mAh} \cdot \mathrm{g}^{-1}\right)$ 和高能量密度 $\left(2600 \mathrm{Wh} \cdot \mathrm{kg}^{-1}\right)$, 是目前 该领域的研究热点 ${ }^{[34]}$. 然而锂硫电池存在以下问题: (1) 单质硫和锂硫化物导电性差, 不利于电池的高倍率性 能. (2)放电过程中电极材料体积会发生膨胀. (3)反应过 程中产生可溶性多硫化物, 导致电极材料循环稳定性降 低, 严重影响了电池的性能. 将硫负载到具有多孔结构 的介孔碳、碳纳米管、有机聚合物等材料中可一定程度 解决多硫化物的溶解流失问题 ${ }^{[35]}$. 在碳材料中掺杂与 多硫化物作用力强的杂元素, 可进一步限制多硫化物的 流失, 然而杂元素掺入量低, 对多硫化物束缚能力有 限 ${ }^{[36]}$. 此外, 金属氧化物或金属硫化物与多硫化物阴离 子和锂离子之间存在协同作用, 也可限制多硫化物的流 失 $^{[34 \mathrm{c}]}$. 与上述材料相比, COFs 作为孔隙率高和设计性 强的多孔材料, 将硫单质负载到 COFs 的纳米孔道中, 不仅可束缚多硫化物的溶解流失, 而且可维持正极微结 构的稳定, 进而增强电极材料的循环稳定性.
Wang 课题组 ${ }^{[37]}$ 首次利用 CTF-1 作为载体负载硫单 质并构筑锂硫电池. 结果表明, CTF-1 对单质硫的装载 量为 $34 \mathrm{wt} \%$, 所构筑的 CTF-1/S 电极在 $168 \mathrm{~mA} \cdot \mathrm{g}^{-1}$ 电 流密度下初始比容量为 $1197 \mathrm{mAh} \cdot \mathrm{g}^{-1}$, 经过 50 次循环 后其比容量仍可维持在 $762 \mathrm{mAh} \cdot \mathrm{g}^{-1}$, 而且表现出了良 好的倍率性能. 该结果首次证明了 COFs 可以作为硫的 载体并构筑锂硫电池. 但是尽管 CTF-1/S 展现出稳定的 比容量和良好的倍率性能, 其比容量以及活性硫的装载 量仍有待提高.

为进一步构筑基于 COFs 的高性能锂硫电池, 该课 题组 ${ }^{[38]}$ 于 2016 年以卟啉为前体制备了 Por-COF，其比表 面积为 $1095 \mathrm{~m}^{2} \cdot \mathrm{g}^{-1}$. 通过装载 $55 \mathrm{wt} \%$ 活性硫所构筑的 Por-COF/S 复合材料在 $840 \mathrm{~mA} \cdot \mathrm{g}^{-1}$ 电流密度下循环 200 次, 比容量仍维持在 $633 \mathrm{mAh} \cdot \mathrm{g}^{-1}$, 其倍率性能相比于 CTF-1/S 有明显提升. 此结果表明, 可通过改变 COFs 结 构制备高性能的锂硫电池. 进一步，该课题组 ${ }^{[39]}$ 于 2018 年合成了具有更高比表面积 $\left(2093 \mathrm{~m}^{2} \cdot \mathrm{g}^{-1}\right)$ 的 Py-COF(如 图 3 所示). 实验结果表明 Py-COF 对硫的装载量高达 70 $\mathrm{wt} \%$, 且 $\mathrm{Py}-\mathrm{COF} / \mathrm{S}$ 表现出了良好的倍率性能，在 8400 $\mathrm{mA} \cdot \mathrm{g}^{-1}$ 电流密度下经过 550 次循环测试后, 其比容量仍 维持在 $481.2 \mathrm{mAh} \cdot \mathrm{g}^{-1}$. 相较于商用的微孔碳材料 BP2000, Py-COF/S 具有更高的比容量. 此外, 为增强 COFs 与活性硫的作用, Wang 课题组 ${ }^{[40]}$ 通过引入功能基 才策略, 合成了氟原子修饰的 FCTF-1. 该 COFs 在负载 了 $53 \mathrm{wt} \%$ 的单质硫后得到 FCTF-1/S 并构筑了锂硫电池. 在 $840 \mathrm{~mA} \cdot \mathrm{g}^{-1}$ 电流密度下经过 150 次循环 FCTF-1/S 能 维持 $833 \mathrm{mAh} \cdot \mathrm{g}^{-1}$ 的稳定比容量.

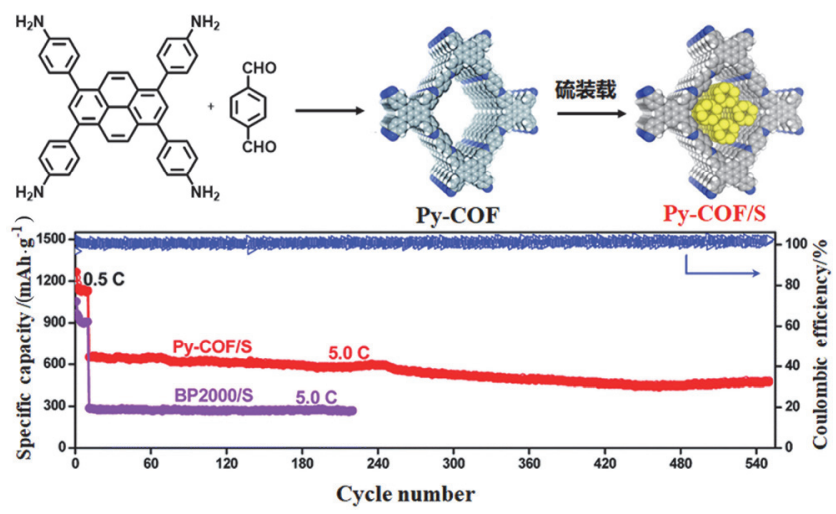

图 $3 \mathrm{Py}-\mathrm{COF} / \mathrm{S}$ 的合成及其在 $8400 \mathrm{~mA} \cdot \mathrm{g}^{-1}$ 电流密度(5.0 C 倍率)下循 环性能

Figure 3 Synthesis of Py-COF/S and its cyclic performance at 8400 $\mathrm{mA} \cdot \mathrm{g}^{-1}$

\section{3 燃料电池}

燃料电池是一种把燃料所具有的化学能直接转换 成电能的化学装置, 具有效率高、污染少的优点, 是最 有发展前途的能源转化技术 ${ }^{[41]}$. 质子传导材料是燃料 电池的重要组成部分. 目前商业化的质子传导材料为具 有优异质子传导率(约 $10^{-1} \mathrm{~S} \cdot \mathrm{cm}^{-1}$ ) 的全氟磺化聚合物 
(Nafion), 但其生产成本高且在高温下工作效率低 ${ }^{[42]}$. 此外, 研究较多的质子传导有机聚合物 ${ }^{[43]}$ 如磺化聚酰 亚胺、磺化聚苯等, 这些聚合物骨架可调控性强易于功 能化, 但其工作温度范围仍较窄 ${ }^{[44]}$. 将可传导质子的客 体分子装入多孔的金属有机框架 (Metal-Organic Frameworks, MOFs) 中, 也可构建质子传导材料 ${ }^{[45]}$, 但 其存在化学稳定性低的问题. 作为一种共价键连接而成 的晶态多孔材料, COFs 具有良好的化学/热稳定性, 且 其有序的孔道为磷酸、磺酸等功能基团引入提供了充足 的空间, 进而作为质子传导材料用于燃料电池的研究.

2014 年, Banerjee 课题组 ${ }^{[46]}$ 合成了含偶氮基元的二 维 $\mathrm{COF}(\mathrm{Tp}-\mathrm{Azo})$, 将其用于磷酸分子的装载得到了 PA-Tp-Azo, 首次探究了 COFs 在质子传导中的应用. 研 究表明, PA-Tp-Azo 表现出了明显的质子传导性能, 在 $98 \%$ 相对湿度 $(\mathrm{RH})$ 和 $332 \mathrm{~K}$ 温度下, 质子传导率为 9.9 $\times 10^{-4} \mathrm{~S} \cdot \mathrm{cm}^{-1}$. 采用相同的策略, Jiang 课题组 ${ }^{[47]}$ 于 2016 年合成了孔径为 $3.26 \mathrm{~nm}$ 的 TPB-DMTP-COF, 并将质子 载体分子三唑和咪唑装载在其孔道中, $130{ }^{\circ} \mathrm{C}$ 下装载三 唑和咪唑的 TPB-DMTP-COF 质子传导率分别为 $1.1 \times$ $10^{-3} \mathrm{~S} \cdot \mathrm{cm}^{-1}$ 和 $4.37 \times 10^{-3} \mathrm{~S} \cdot \mathrm{cm}^{-1} .2016$ 年 Banerjee 课题 组 ${ }^{[48]}$ 利用双通道质子传导策略: 一方面通过磺酸基团 在 COFs 骨架上直接构筑内部质子传导通道; 另一方面 利用骨架上的吡啶基团在孔道中引入植酸分子作为外 部质子传导通道, 探究了高温无水条件下 COFs 在质子 传导中的应用. 为进一步构筑具有较高质子传导率且可 简单制备质子交换膜的 COFs，2018 年 Banerjee 课题 组 ${ }^{[49]}$ 选择既可作为催化剂又可作为质子传导介质的对 甲苯磺酸, 制备了在 $80{ }^{\circ} \mathrm{C}$ 和 $95 \%(\mathrm{RH})$ 下质子传导率高 达 $7.8 \times 10^{-2} \mathrm{~S} \cdot \mathrm{cm}^{-1}$ 的 PTSA@TpAzo 薄膜, 并将该膜用 于燃料电池的研究. 结果表明, 所组装的燃料电池极化 后最高输出功率达到 $24 \mathrm{~mW} \cdot \mathrm{cm}^{-2}$, 为目前 $\mathrm{COF}$ 材料中 报道的最高值.

\section{4 超级电容器}

与二次电池相比, 超级电容器具有更高的功率密 度, 可实现短时间内的快速充放电. 超级电容器的存储 容量通常与电极的比表面积和活性组分的氧化还原反 应有关 ${ }^{[50]}$. 多孔碳是目前研究较多的电容器材料, 这类 材料比表面积高, 但其离子传输能力和循环性能差 ${ }^{[51]}$. 此外, 一些导电聚合物如聚乙炔、聚苯胺、聚吡咯也被 应用于电容器的研究 ${ }^{[52]}$. 然而, 这些聚合物在充放电过 程中会产生膨胀与收缩, 循环稳定性差. COFs 作为一种 比表面积高和可设计性强的新型有机多孔材料, 具有刚 性的骨架结构和有序的离子传输通道, 在超级电容器中 具有广阔的应用前景.

2013 年, Dichtel 课题组 ${ }^{[53]}$ 首次研究了 COF 在超级 电容器中的应用. 他们以二氨基蒽醌为前体设计合成了 DAAQ-TFP-COF, 该 COF 在 $100 \mathrm{~mA} \cdot \mathrm{g}^{-1}$ 的电流密度下, 经过 10 次循环后表现出 $40 \pm 9 \mathrm{~F}^{\circ} \mathrm{g}^{-1}$ 的电容值, 且 5000
次循环后其电容值没有明显下降. 然而, 由于电极内部 COFs 颗粒的取向具有随机性，该 COF 中只有 $2.5 \%$ 的活 性单元发挥作用. 为提高 COFs 框架中活性单元的利用 率，该课题组 ${ }^{[54]}$ 于 2015 年在金基底上生长出了取向均 一的 DAAQ-TFP-COF 薄膜 (图 4 所示), 与其粉末样品 相比电容值提高了 4 倍, 且活性单元利用率增加到 $80 \% \sim 99 \%$. 为进一步提高电容器性能, 该课题组 ${ }^{[55]}$ 于 2016 年通过电聚合方式将聚乙烯二氧噻吩(PEDOT)生 长在 DAAQ-TFP-COF 薄膜的孔道中, PEDOT 和 DAAQ 单元之间的协同作用可提高 DAAQ-TFP-COF 薄膜的导 电性和电容量. 通过循环伏安测试和恒电流充放电测 试, PEDOT-DAAQ-TFP-COF 表现出更优异的电容性质. 且 PEDOT-DAAQ-TFP-COF 具有非常高的稳定性, 在高 充电频率下经过 10000 次循环仍保持较高的电容量.

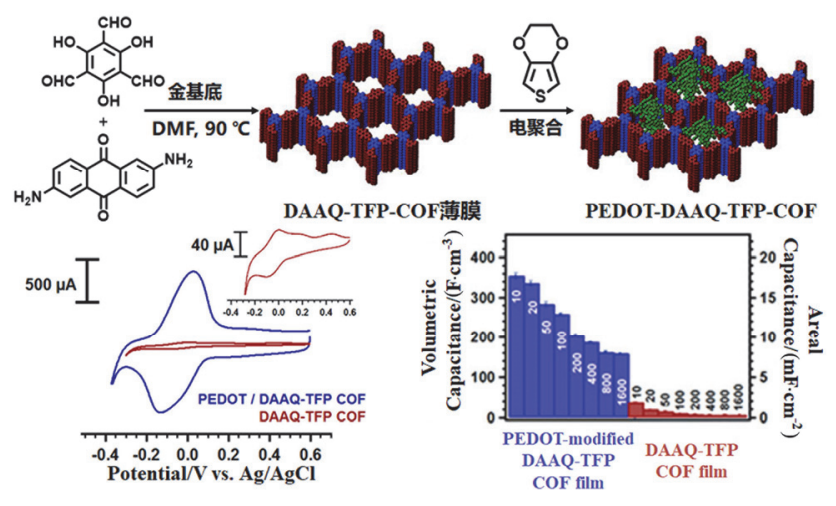

图 4 PEDOT-DAAQ-TFP-COF 的合成及其电容性能表征 Figure 4 Synthesis of PEDOT-DAAQ-TFP-COF and its charge storage performance

此外, 引入不同的功能基团也可调节 COFs 的电容 性质. 2015 年 Jiang 课题组 ${ }^{[56]}$ 通过后合成修饰策略, 利 用点击反应将四甲基哌啶氮氧化物 (TEMPO)修饰到 NiP-COF 框架上, 合成了一系列[TEMPO $]_{X \%}-\mathrm{NiP}-\mathrm{COFs}$. 由于 TEMPO 可发生可逆的氧化还原反应, [TEMPO $]_{X \%}-$ $\mathrm{NiP}-\mathrm{COF}$ 表现出较高的电容量. 其中 [TEMPO $]_{100 \%}-$ $\mathrm{NiP}-\mathrm{COF}$ 在 $100 \mathrm{~mA} \cdot \mathrm{g}^{-1}$ 电流密度下电容值为 $167 \mathrm{~F} \bullet \mathrm{g}^{-1}$, 将电流密度提升至 $2000 \mathrm{~mA} \cdot \mathrm{g}^{-1}$ 时电容值仍保持在 113 $\mathrm{F} \bullet \mathrm{g}^{-1}$. Banerjee 课题组 ${ }^{[57]}$ 于 2017 年将苯酚引入到 COFs 中合成了 $\mathrm{TpPa}-(\mathrm{OH})_{2}-\mathrm{COF}$, 在 $500 \mathrm{~mA} \cdot \mathrm{g}^{-1}$ 的电流密度 下, $\mathrm{TpPa}-(\mathrm{OH})_{2}-\mathrm{COF}$ 的电容值高达 $416 \mathrm{~F} \cdot \mathrm{g}^{-1}$; 在 5000 $\mathrm{mA} \cdot \mathrm{g}^{-1}$ 电流密度下, 经过 10000 次循环测试后该 $\mathrm{COF}$ 电容量仍能够保持初始容量的 $66 \%$.

\section{COFs 在能源转化中的应用}

人类的生活离不开能源, 其中化石能源的开发和利 用满足了人类对于工业生产和日常生活的绝大部分能 源需求. 然而, 化石能源储量有限, 且消耗过程中会带 来严重的环境污染问题，因此开发高效、可循环使用的 
新能源成为了目前科研工作者的研究重点 ${ }^{[13]}$. 在催化 剂作用下, 将电能或者光能存储在化学键中转化为化学 能, 制备一系列如 $\mathrm{H}_{2} 、 \mathrm{CO} 、 \mathrm{CH}_{3} \mathrm{OH}$ 等气态或液态燃料, 是发展新能源的有效手段 ${ }^{[58]}$. 基于 COFs 固有的结构特 点和自下而上的设计策略, 研究者们可选择不同的功能 基元和具有较高稳定性的连接键构筑高效稳定的催化 剂. 本部分将分别介绍 COFs 在水分解反应和 $\mathrm{CO}_{2}$ 还原 反应中的应用.

\section{1 水分解反应}

通过水分解反应的两个半反应一一析氢反应 (Hydrogen Evolution Reaction, HER)和析氧反应(Oxygen Evolution Reaction, OER), 可将光能或电能转化为化学 能存储在 $\mathrm{H}-\mathrm{H}$ 键或 $\mathrm{O}-\mathrm{O}$ 键中. 为实现这一能量转化 过程, 常需加入合适的催化剂 ${ }^{[10 f, 59]}$. 相比于其他催化 剂, COFs 材料在水分解反应中具有独特的优势. 一方 面, COFs 层间的 $\pi$ 共轭结构有利于激发态电子传输到催 化剂表面发生反应, 提高催化效率. 另一方面, 将不同 的功能基团引入至 COFs 骨架中, 可实现 COFs 催化性 能的有效调控.

自 1972 年 Honda 和 Fujishima ${ }^{[60]}$ 首次将 $\mathrm{TiO}_{2}$ 用于 光催化产氢以来, 无机半导体如金属氧化物、硫氧化物、 氮氧化物等, 用于构筑光催化剂 ${ }^{[61]}$. 与无机半导体相 比, 有机半导体如碳氮化合物 ${ }^{[62]}$ 、共轭微孔聚合物 ${ }^{[63]}$ 、 线性共轭聚合物 ${ }^{[64]}$, 半导体性能调控性强, 但此类材料 结晶性差, 难以对其构效关系进行深入研究. COFs 作为 一类比表面积高、可设计性强的晶态有机多孔材料, 在 光催化产氢领域中具有巨大潜力. 2014 年, Lotsch 课题 组 ${ }^{[65]}$ 以三嗪为功能单元合成了 TFPT-COF, 首次实现了 COFs 在光解水领域的应用. 随后, 为进一步探究嗪类 分子作为功能基元对光解水催化效果的影响, 该课题 组 ${ }^{[66]}$ 设计并合成了一系列含嗪类分子的 COFs. 研究表 明, 从含 1 个氮原子的吡啶结构到含 3 个氮原子的三嗪 环结构, 光催化产氢速率从 $90 \mu \mathrm{mol} \cdot \mathrm{h}^{-1} \cdot \mathrm{g}^{-1}$ 提升至 1703 $\mu \mathrm{mol} \bullet \mathrm{h}^{-1} \cdot \mathrm{g}^{-1}$, 可能原因是嗪类分子中氮原子数量的增 加, 使嗪类分子的平面性逐渐增强, 所构筑的 COFs 拥 有更加长程有序的结构. 2018 年, Schmidt 课题组 ${ }^{[67]}$ 合成 了含乙炔结构的 TP-EDDA-COF 和丁二炔结构的 TP-BDDA-COF. 研究表明, 相比于 TP-EDDA-COF, TP-BDDA-COF 光催化产氢速率由 $30 \pm 5 \mu \mathrm{mol} \cdot \mathrm{h}^{-1} \cdot \mathrm{g}^{-1}$ 提升至 $324 \pm 10 \mu \mathrm{mol} \cdot \mathrm{h}^{-1} \cdot \mathrm{g}^{-1}$, 证明了炔基功能基元在 光催化反应中的重要性. 同年, Cooper 课题组 ${ }^{[68]}$ 将苯并 噻吩砜引入 COFs 骨架结构中构筑了 S-COF 和 FS-COF(如图 5 所示), 这两种 COFs 都展现出极高的光 催化活性, 其产氢速率分别为 $4.44 \mathrm{mmol} \cdot \mathrm{h}^{-1} \cdot \mathrm{g}^{-1}$ 和 10.1 $\mathrm{mmol} \cdot \mathrm{h}^{-1} \cdot \mathrm{g}^{-1}$. 此外, 通过染料敏化后的 FS-COF 展现 出目前 COFs 材料中最高的光催化产氢速率 (16.3 $\left.\mathrm{mmol} \cdot \mathrm{h}^{-1} \cdot \mathrm{g}^{-1}\right)$.

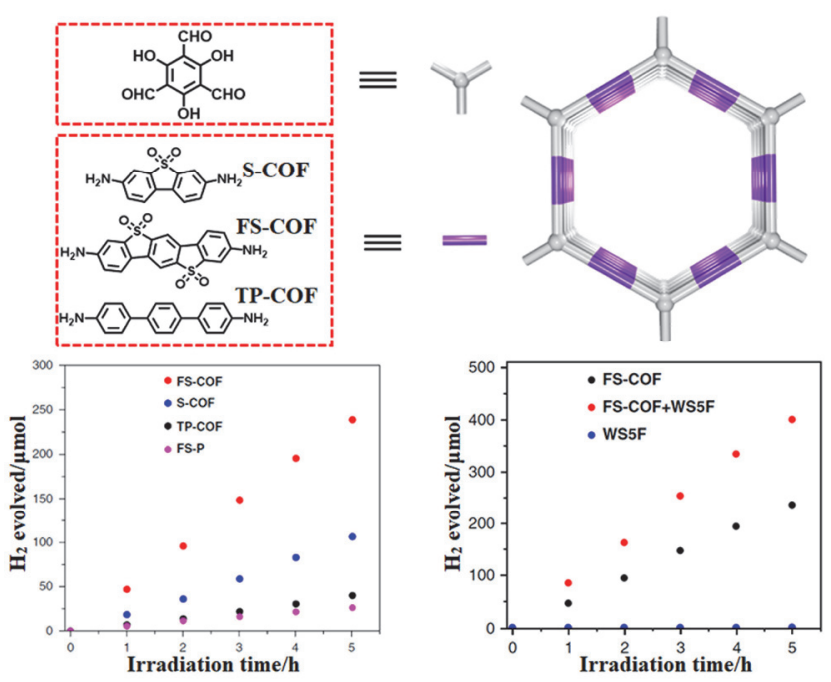

图 5 S-COF、FS-COF 和 TP-COF 的构筑及其光催化产氢性能 Figure 5 Construction of S-COF, FS-COF and TP-COF and their photocatalysis performance

相对于 HER 反应, OER 反应涉及到更为复杂的四 电子转移过程 ${ }^{[69]}$, 可通过电催化手段来实现. 常用的 OER 催化剂有过渡金属氧化物如 $\mathrm{RuO}_{2}$ 和 $\mathrm{IrO}_{2}$, 具有优 异的催化性能, 但其储量低和成本高限制了其广泛应 用 ${ }^{[70]}$. 金属钴作为一类储量丰富的元素，其卟啉、酞菁 等配合物常被用于 OER 催化研究 ${ }^{[71]}$, 然而这类催化剂 在使用过程中会发生降解, 使其催化性能降低 ${ }^{[72]}$. COFs 是一类可调控性强的框架材料, 不仅可实现活性位点的 精确调控, 而且可作为异相催化剂实现材料的循环利 用. 2016 年 Kurungot 课题组 ${ }^{[73]}$ 将 $\mathrm{Co}^{2+}$ 修饰到含有联吡 啶功能基元的 TpBpy-COF 骨架上, 制备了 Co-TpBpyCOF 并将其用于 OER 研究. 实验表明, 在 $1 \mathrm{~mA} \cdot \mathrm{cm}^{-2}$ 的电流密度下 Co-TpBpy-COF 的过电位为 $400 \mathrm{mV}$, 塔菲 尔斜率为 $59 \mathrm{mV} \cdot \mathrm{dec}^{-1}$. 线性扫描伏安法(LSV)的结果显 示, 1000 次扫描后其 OER 活性仍能保持初始值的 94\%, 且在催化水解过程中具有良好的循环稳定性, 证明过渡 金属元素与 COFs 的结合能够形成高效稳定的催化剂. 此外，通过装载过渡金属纳米颗粒，也可制备高性能催 化剂. Vaidhyanathan 课题组 ${ }^{[74]}$ 同年以三苯胺为前体合成 了 IISERP-COF2, 该 COF 以 $\mathrm{N}$ 为节点形成了柔性的 $\mathrm{COF}$ 骨架, 可用以装载 $\mathrm{Ni}_{x} \mathrm{Co}_{y}(\mathrm{OH})_{2}$ 纳米颗粒, $\mathrm{COF}$ 与纳 米颗粒所形成的复合物在 $10 \mathrm{~mA} \cdot \mathrm{cm}^{-2}$ 的电流密度下表 现出过电位为 $258 \mathrm{mV}$, 塔菲尔斜率为 $38.9 \mathrm{mV} \cdot \mathrm{dec}^{-1}$. 进一步, 该课题组 ${ }^{[75]}$ 利用能带更窄的 IISERP-COF3 装 载 $\mathrm{NiN}_{3}$ 纳米颗粒, 所合成复合物在 $10 \mathrm{~mA} \cdot \mathrm{cm}^{-2}$ 的电流 密度下过电位为 $230 \mathrm{mV}$, 相比 IISERP-COF3 本身的过 电位明显降低. 作为催化剂, 该复合物产氧效果显著, 产氧率为 $230 \mathrm{mmol} \cdot \mathrm{h}^{-1} \cdot \mathrm{g}^{-1}$.

\section{$3.2 \mathrm{CO}_{2}$ 还原反应}

利用光能或电能将 $\mathrm{CO}_{2}$ 转化为燃料和化学品, 不仅 
可缓解温室效应, 而且可实现能源转化. 其中, 催化 $\mathrm{CO}_{2}$ 还原为 $\mathrm{CO}$ 是最具有应用前景的 $\mathrm{CO}_{2}$ 转化技术之 - ${ }^{[76]}$. COFs 作为一类结构可调的晶态多孔材料, 可作为 $\mathrm{CO}_{2}$ 还原催化剂. 首先, $\mathrm{COFs}$ 的多孔性有利于 $\mathrm{CO}_{2}$ 的吸 附和扩散; 其次, 通过活性中心的引入及其空间排布的 精确调控, 可对 COFs 骨架进行功能化修饰; 最后, 调 控 COFs 骨架的电子传输性能, 可改变 COFs 的催化性 能.

光催化还原 $\mathrm{CO}_{2}$ 是利用太阳能获得清洁能源的有 效手段. 许多无机半导体如 $\mathrm{TiO}_{2} 、 \mathrm{CdS} 、 \mathrm{ZnGa}_{2} \mathrm{O}_{4}$ 等, 可 作为催化剂还原 $\mathrm{CO}_{2}$, 但其可见光吸收能力差, 催化 $\mathrm{CO}_{2}$ 还原效率低 ${ }^{[77]}$. 此外, 分子篮也可用于 $\mathrm{CO}_{2}$ 还原催 化剂的研究, 然而其可调控性差大大限制了其在光催化 领域的应用 ${ }^{[78]}$. COFs 骨架结构稳定且易于光活性基团 的引入, 在光催化 $\mathrm{CO}_{2}$ 还原领域有巨大的应用前景. 2018 年 Huang 课题组 ${ }^{[79]}$ 以三嗪和联吡啶为前体合成了 具有光活性的二维 COF, 进一步通过后合成修饰将 $\mathrm{Re}$ 配合物 $\left(\mathrm{Re}(\mathrm{bpy})(\mathrm{CO})_{3} \mathrm{Cl}\right)$ 配位至 $\mathrm{COF}$ 骨架上, 构筑了 $\mathrm{Re}-\mathrm{COF}$ 催化剂用于光催化 $\mathrm{CO}_{2}$ 还原. 结果表明, 在可 见光照射 $20 \mathrm{~h}$ 后每克 Re-COF 催化剂产生约 $15 \mathrm{mmol}$ $\mathrm{CO}$, 而且具有高选择性(98\%).

电催化也是高效催化还原 $\mathrm{CO}_{2}$ 的常用方法. 2015 年 Yaghi 课题组 ${ }^{[80]}$ 利用钴卟啉合成了 COF-366-Co, 在催化 还原 $\mathrm{CO}_{2}$ 的实验中(如图 6 所示)表现出高达 $90 \%$ 的法拉 第效率和 34000 的 $\mathrm{TON}_{\mathrm{EA}}$ 值(基于电活性 $\mathrm{Co}$ ). 相比于钴 卟啉前体其催化活性提高了 26 倍, 且在 $24 \mathrm{~h}$ 后仍能维 持较高的催化效率. 为增加 COFs 中活性位点和反应物 分子之间的接触, 他们将前体对苯二甲醛换为联苯二甲 醛合成了孔径更大的 COF-367-Co, 经过 $24 \mathrm{~h}$ 的催化还 原 $\mathrm{CO}_{2}$ 试验, 每克催化剂产生 $4.5 \mathrm{mmol} \mathrm{CO}$, 其 $\mathrm{TON}_{\mathrm{EA}}$ 提升至 48000. 此外, 通过引入推拉电子基团可对整个
COFs 框架的电子传输进行调控，进而实现骨架中活性 中心催化性能的调控. 2018 年，该课题组 ${ }^{[81]}$ 将具有不同 的推拉电子效应的基团引入 COF-366-Co 的骨架, 通过 改变催化活性中心的电子特性, 制备了一系列对 $\mathrm{CO}_{2}$ 还 原具有不同催化性能的催化剂.

\section{4 结论与展望}

近年来, 二维 COFs 因其固有结构特点, 在能源存 储和能源转化领域的应用研究取得了一系列进展. 本文 主要综述了 COFs 作为电极材料在二次电池(锂离子电 池、锂硫电池、燃料电池) 以及超级电容器方面和作为 催化剂及催化剂载体在水分解反应以及 $\mathrm{CO}_{2}$ 还原反应 方面的研究进展. 与其他材料如金属氧化物、碳纳米材 料、有机聚合物等相比, COFs 孔道规整、孔径可调、比 表面积高、可设计性强, 在材料性能的优化和构效关系 的探究等方面具有独特的优势. 例如, 通过前体的设计, COFs 骨架上可引入不同的官能团，从而调控催化位点 的活性, 提升 COFs 在电催化 $\mathrm{CO}_{2}$ 还原中的性能. 但不 可否认，作为一种正处于发展阶段的新型有机多孔材 料, COFs 在能源领域中的研究仍存在较多问题待解决: (1)如何大规模生产低成本 COFs? 当前 COFs 主要通过 溶剂热等方法合成，难以实现其大规模制备. 而且， COFs 合成的成本较高, 极大限制了其在能源领域中的 进一步应用. 因而开发简单高效的 COFs 制备方法是研 究者们下一步亟待解决的问题. (2)如何提高 COFs 的导 电性? 尽管和碳纳米管等材料进行复合可有效提高电 极的导电性，但 COFs 本身导电性仍处于绝缘体和半导 体范围. 为提高 COFs 的导电性, 可选择具有大 $\pi$ 共轭 结构的分子作为基元，同时选择共轭程度更高的连接方 式; 另外提高 COFs 的结晶度, 减少材料中的缺陷，也

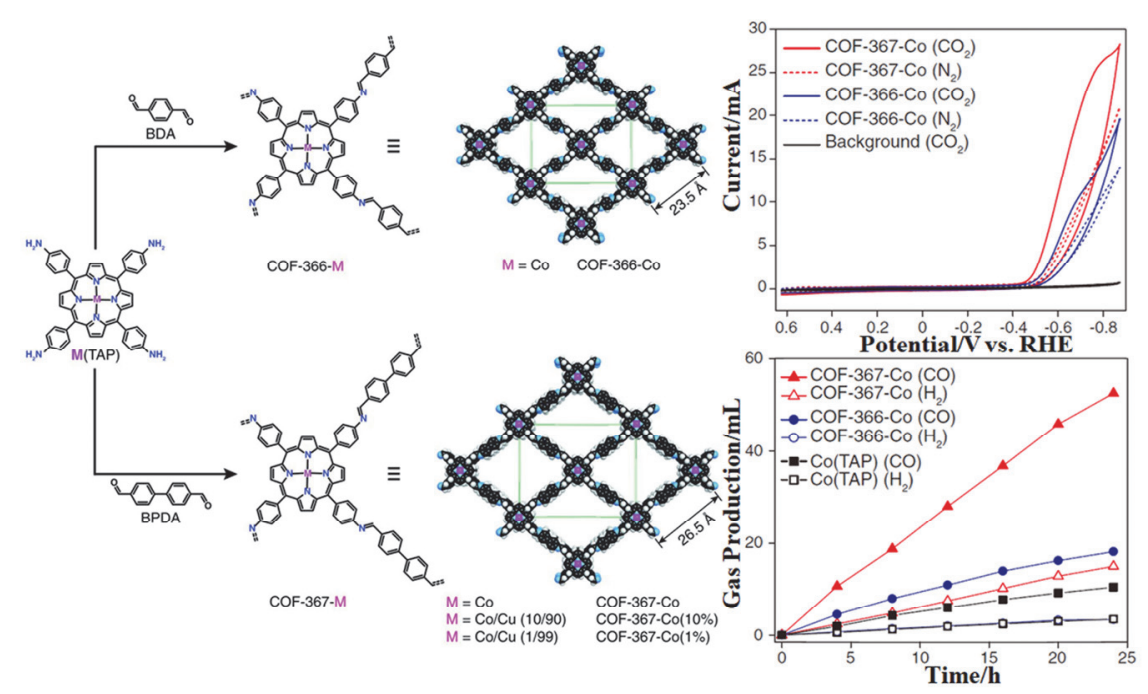

图 6 COF-366-Co 和 COF-367-Co 的合成(a)、循环伏安曲线(b)及产 $\mathrm{CO}$ 性能(c)

Figure 6 (a) Syntheses of COF-366-Co, COF-367-Co, (b) cyclic voltammograms of COF-366-Co, COF-367-Co and (c) the volume of CO produced by COF-366-Co, COF-367-Co 
有利于提高 COFs 的导电性. (3)如何进一步提高 COFs 在能源存储与能源转化领域中的性能? 如在超级电容 器的应用中, 虽然 COFs 电容量已达到较高水平, 但与 目前研究较为广泛金属氧化物相比仍有待提高. 此外, 提高 COFs 的比表面积和孔径也有可能提升 COFs 在能 源领域的性能.

总之, 作为一种具有潜力的新型晶态有机多孔材 料, COFs 在能源领域具有较大的发展空间. 为使 COFs 在能源领域进一步发展, 一方面应研究清楚 COFs 的生 长机理, 发展出更绿色、更简单、更高效的制备方法, 实 现 COFs 的批量生产; 另一方面, 进一步拓展功能基元 并将其引入至 COFs, 旨在改善 COFs 的部分劣势如导电 性差等问题, 促进 COFs 领域与能源领域的共同发展. 我们相信, 随着 COFs 研究的不断深入, 将有更多低成 本、高性能的 COFs 应用于能源领域, 并推动该领域的 发展.

\section{作者简介}

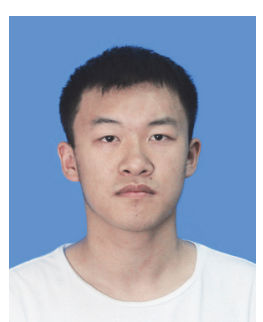

彭正康, 本科毕业于北京化工大学, 现为武汉大学化学 与分子科学硕士生, 目前研究方向为共价有机框架的构筑及 其电化学性质的探究.

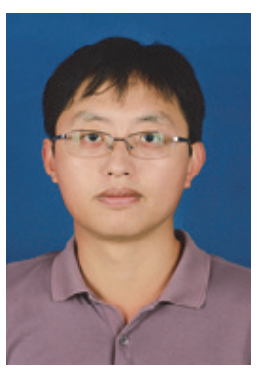

汪成, 2003 年获武汉大学学士学位, 2008 年在中国科学院 化学研究所获理学博士学位, 随后在美国西北大学化学系进 行博士后研究. 2012 年 4 月加入武汉大学化学与分子科学学院, 任教授、博士生导师. 2013 年被聘为湖北省 “楚天学者” 特聘 教授, 2015 年获得湖北省杰出青年基金资助, 2019 年入选第四 批 “万人计划” 青年拔尖人才. 主要从事多孔框架晶体材料(包 括 COFs 和 MOFs) 的研究.

\section{References}

[1] (a) Das, S.; Heasman, P.; Ben, T.; Qiu, S. Chem. Rev, 2017, 117, 1515. (b) Huang, N.; Wang, P.; Jiang, D. Nat. Rev. Mater. 2016, 1 , 16068. (c) Waller, P. J.; Gandara, F.; Yaghi, O. M. Acc. Chem. Res. 2015, 48, 3053. (d) Ding, S. Y.; Wang, W. Chem. Soc. Rev. 2013, 42, 548.

[2] (a) Zhou, B.; Chen, L. Acta Chim. Sinica 2015, 73, 487. (周宝龙, 陈龙，化学学报, 2015, 73, 487.) (b) Xu, Y.; Jin, S.; Xu, H.; Nagai,
A.; Jiang, D. Chem. Soc. Rev. 2013, 42, 8012. (c) Cooper, A. I. $A d v$. Mater. 2009, 21, 1291.

[3] (a) Ren, H.; Zhu, G. Acta Chim. Sinica 2015, 73, 587. (任浩, 朱广 山, 化学学报, 2015, 73, 587.) (b) Ben, T.; Qiu, S. CrystEngComm 2013, 15, 17. (c) Ben, T.; Pei, C.; Zhang, D.; Xu, J.; Deng, F.; Jing, X.; Qiu, S. Energy Environ. Sci. 2011, 4, 3991. (d) Ben, T.; Ren, H.; Ma, S.; Cao, D.; Lan, J.; Jing, X.; Wang, W.; Xu, J.; Deng, F.; Simmons, J. M.; Qiu, S.; Zhu, G. Angew. Chem. Int. Ed. 2009, 48, 9457.

[4] (a) Tan, L.; Tan, B. Acta Chim. Sinica 2015, 73, 530. (谭良骁, 谭必 恩，化学学报, 2015, 73, 530.) (b) Huang, J.; Turner, S. R. Polym. Rev. 2017, 58, 1. (c) Tan, L.; Tan, B. Chem. Soc. Rev. 2017, 46, 3322.

[5] Côté, A. P.; Benin, A. I.; Ockwig, N. W.; O'Keeffe, M.; Matzger, A. J.; Yaghi, O. M. Science 2005, 310, 1166.

[6] (a) Zeng, Y.; Zou, R.; Zhao, Y. Adv. Mater. 2016, 28, 2855. (b) Kang, Z.; Peng, Y.; Qian, Y.; Yuan, D.; Addicoat, M. A.; Heine, T.; Hu, Z.; Tee, L.; Guo, Z.; Zhao, D. Chem. Mater. 2016, 28, 1277. (c) Song, J. R.; Sun, J.; Liu, J.; Huang, Z. T.; Zheng, Q. Y. Chem. Commun. 2014, 50, 788. (d) Zhou, T. Y.; Xu, S. Q.; Wen, Q.; Pang, Z. F.; Zhao, X. J. Am. Chem. Soc. 2014, 136, 15885.

[7] (a) Ding, S. Y.; Dong, M.; Wang, Y. W.; Chen, Y. T.; Wang, H. Z.; Su, C. Y.; Wang, W. J. Am. Chem. Soc. 2016, 138, 3031. (b) Wang, P.; Zhou, F.; Zhang, C.; Yin, S. Y.; Teng, L.; Chen, L.; Hu, X. X.; Liu, H. W.; Yin, X.; Zhang, X. B. Chem. Sci. 2018, 9, 8402. (c) Dalapati, S.; Jin, E.; Addicoat, M.; Heine, T.; Jiang, D. J. Am. Chem. Soc. 2016, 138, 5797. (d) Lin, G.; Ding, H.; Yuan, D.; Wang, B.; Wang, C. J. Am. Chem. Soc. 2016, 138, 3302. (e) Zhu, M. W.; Xu, S. Q.; Wang, X. Z.; Chen, Y.; Dai, L.; Zhao, X. Chem. Commun. 2018, 54, 2308. (f) Yang, T.; Cui, Y.; Chen, H.; Li, W. Acta Chim. Sinica 2017, 75, 339. (杨涛, 崔亚男, 陈怀银, 李伟华, 化学学报, 2017, 75,339 .)

[8] (a) Ding, S. Y.; Gao, J.; Wang, Q.; Zhang, Y.; Song, W. G.; Su, C. Y.; Wang, W. J. Am. Chem. Soc. 2011, 133, 19816. (b) Fang, Q.; Gu, S.; Zheng, J.; Zhuang, Z.; Qiu, S.; Yan, Y. Angew. Chem. Int. Ed. 2014 53, 2878. (c) Lu, S.; Hu, Y.; Wan, S.; McCaffrey, R.; Jin, Y.; Gu, H.; Zhang, W. J. Am. Chem. Soc. 2017, 139, 17082. (d) Zhang, J.; Han, X.; Wu, X.; Liu, Y.; Cui, Y. J. Am. Chem. Soc. 2017, 139, 8277. (e) Wei, P. F.; Qi, M. Z.; Wang, Z. P.; Ding, S. Y.; Yu, W.; Liu, Q.; Wang, L. K.; Wang, H. Z.; An, W. K.; Wang, W. J. Am. Chem. Soc. 2018, 140, 4623. (f) Chen, R.; Shi, J. L.; Ma, Y.; Lin, G.; Lang, X.; Wang, C. Angew. Chem. Int. Ed. 2019, 58, 6430.

[9] (a) Spitler, E. L.; Dichtel, W. R. Nat. Chem. 2010, 2, 672. (b) Ding, H.; Li, J.; Xie, G.; Lin, G.; Chen, R.; Peng, Z.; Yang, C.; Wang, B.; Sun, J.; Wang, C. Nat. Commun. 2018, 9, 5234. (c) Feng, X.; Liu, L.; Honsho, Y.; Saeki, A.; Seki, S.; Irle, S.; Dong, Y.; Nagai, A.; Jiang, D. Angew. Chem. Int. Ed. 2012, 51, 2618. (d) Sun, B.; Zhu, C.-H.; Liu, Y.; Wang, C.; Wan, L.-J.; Wang, D. Chem. Mater. 2017, 29, 4367. (e) Medina, D. D.; Sick, T.; Bein, T. Adv. Energy Mater. 2017, 7,1700387

[10] (a) Ma, L.; Wang, S.; Feng, X.; Wang, B. Chin. Chem. Lett. 2016, 27, 1383. (b) Alahakoon, S. B.; Thompson, C. M.; Occhialini, G.; Smaldone, R. A. ChemSusChem 2017, 10, 2116. (c) Mandal, A. K.; Mahmood, J.; Baek, J.-B. ChemNanoMat 2017, 3, 373. (d) Medina, D. D.; Sick, T; Bein, T. Adv. Energy Mater. 2017, 7, 1700387. (e) Zhan, X.; Chen, Z.; Zhang, Q. J. Mater. Chem. A 2017, 5, 14463. (f) Banerjee, T.; Gottschling, K.; Savasci, G.; Ochsenfeld, C.; Lotsch, B. V. ACS Energy Lett. 2018, 3, 400. (g) Lin, C. Y.; Zhang, D.; Zhao, Z.; Xia, Z. Adv. Mater. 2018, 30, 1703646. (h) Cao, S.; Li, B.; Zhu, R.; Pang, H. Chem. Eng. J. 2019, 355, 602. (i) Wan, G.; Fu, Y.; Guo, J.; Xiang, Z. Acta. Chim. Sinica 2015, 73, 557. (万钢, 付宇昂, 郭 佳宁，向中华，化学学报, 2015, 73, 557.)

[11] (a) Feng, X.; Chen, L.; Honsho, Y.; Saengsawang, O.; Liu, L.; Wang, L.; Saeki, A.; Irle, S.; Seki, S.; Dong, Y.; Jiang, D. Adv. Mater. 2012, 24, 3026. (b) Chen, X.; Addicoat, M.; Irle, S.; Nagai, A.; Jiang, D. J. Am. Chem. Soc. 2013, 135, 546. (c) Colson, J. W.; Dichtel, W. R. Nat. Chem. 2013, 5, 453. (d) Yang, L.; Wei, D.-C. Chin. Chem. Lett. 2016, 27, 1395.

[12] (a) Lohse, M. S.; Stassin, T.; Naudin, G.; Wuttke, S.; Ameloot, R.; De Vos, D.; Medina, D. D.; Bein, T. Chem. Mater. 2016, 28, 626. (b) Waller, P. J.; Lyle, S. J.; Osborn Popp, T. M.; Diercks, C. S.; Reimer, J. A.; Yaghi, O. M. J. Am. Chem. Soc. 2016, 138, 15519. (c) Zhuang, X.; Zhao, W.; Zhang, F.; Cao, Y.; Liu, F.; Bi, S.; Feng, X. Polym. Chem. 2016, 7, 4176. (d) Jin, E.; Asada, M.; Xu, Q.; Dalapati, S.; Addicoat, M. A.; Brady, M. A.; Xu, H.; Nakamura, T.; Heine, T.; Chen, Q.; Jiang, D. Science 2017, 357, 673. (e) Li, X.; Zhang, C.; Cai, S.; Lei, X.; Altoe, V.; Hong, F.; Urban, J. J.; Ciston, J.; Chan, E. 
M.; Liu, Y. Nat. Commun. 2018, 9, 2998. (f) Han, X.; Huang, J.; Yuan, C.; Liu, Y.; Cui, Y. J. Am. Chem. Soc. 2018, 140, 892. (g) Zhang, B.; Wei, M.; Mao, H.; Pei, X.; Alshmimri, S. A.; Reimer, J. A.; Yaghi, O. M. J. Am. Chem. Soc. 2018, 140, 12715.

[13] Chu, S.; Cui, Y.; Liu, N. Nat. Mater. 2016, 16, 16

[14] (a) Zhu, J.; Yang, D.; Yin, Z.; Yan, Q.; Zhang, H. Small 2014, 10, 3480. (b) Zhang, Q.; Uchaker, E.; Candelaria, S. L.; Cao, G. Chem. Soc. Rev. 2013, 42, 3127. (c) Aricò, A. S.; Bruce, P.; Scrosati, B.; Tarascon, J.-M.; Schalkwijk, W. Nat. Mater. 2005, 4, 366.

[15] Hu, L. H.; Wu, F. Y.; Lin, C. T.; Khlobystov, A. N.; Li, L. J. Nat. Commun. 2013, 4, 1687.

[16] (a) Whittingham, M. S. Chem. Rev. 2004, 104, 4271. (b) Ellis, B. L.; Lee, K. T.; Nazar, L. F. Chem. Mater. 2010, 22, 691. (c) Goodenough, J. B.; Kim, Y. Chem. Mater. 2010, 22, 587.

[17] (a) Liang, Y.; Tao, Z.; Chen, J. Adv. Energy Mater. 2012, 2, 742. (b) Song, Z.; Zhou, H. Energy Environ. Sci. 2013, 6, 2280. (c) Nishida, S.; Yamamoto, Y.; Takui, T.; Morita, Y. ChemSusChem 2013, 6, 794. (d) Armand, M.; Grugeon, S.; Vezin, H.; Laruelle, S.; Ribiere, P.; Poizot, P.; Tarascon, J. M. Nat. Mater. 2009, 8, 120. (e) Armand, M.; Tarascon, J.-M. Nature 2008, 451, 652. (f) He, Q.; Zhang, C.; Li, X.; Wang, X.; Mu, P.; Jiang, J. Acta Chim. Sinica 2018, 76, 202. (贺倩, 张崇, 李晓, 王雪, 牟攀, 蒋加兴, 化学学报, 2018, 76, 202.)

[18] (a) Mike, J. F.; Lutkenhaus, J. L. ACS Macro Lett. 2013, 2, 839. (b) Yang, Y.; Wang, C.; Yue, B.; Gambhir, S.; Too, C. O.; Wallace, G. G. Adv. Energy Mater. 2012, 2, 266.

[19] (a) Wu, H.; Shevlin, S. A.; Meng, Q.; Guo, W.; Meng, Y.; Lu, K.; Wei, Z.; Guo, Z. Adv. Mater. 2014, 26, 3338. (b) Song, Z.; Qian, Y.; Liu, X.; Zhang, T.; Zhu, Y.; Yu, H.; Otani, M.; Zhou, H. Energy Environ. Sci. 2014, 7, 4077. (c) Song, Z.; Zhan, H.; Zhou, Y. Angew. Chem. Int. Ed. 2010, 49, 8444. (d) Armand, M.; Grugeon, S.; Vezin, H.; Laruelle, S.; Ribiere, P.; Poizot, P.; Tarascon, J. M. Nat. Mater. 2009, 8, 120. (e) Chen, H.; Armand, M.; Courty, M.; Jiang, M.; Grey, C. P.; Dolhem, F.; Tarascon, J.-M.; Poizot, P. J. Am. Chem. Soc. 2009, 131, 8984.

[20] (a) Zhan, L.; Song, Z.; Zhang, J.; Tang, J.; Zhan, H.; Zhou, Y.; Zhan, C. Electrochim. Acta 2008, 53, 8319. (b) Zhang, J. Y.; Kong, L. B.; Zhan, L. Z.; Tang, J.; Zhan, H.; Zhou, Y. H.; Zhan, C. M. J. Power Sources 2007, 168, 278.

[21] (a) Jähnert, T.; Hager, M. D.; Schubert, U. S. J. Mater. Chem. A 2014, 2, 15234. (b) Janoschka, T.; Hager, M. D.; Schubert, U. S. Adv. Mater. 2012, 24, 6397. (c) Nakahara, K.; Oyaizu, K.; Nishide, H. Chem. Lett. 2011, 40, 222. (d) Morita, Y.; Nishida, S.; Murata, T.; Moriguchi, M.; Ueda, A.; Satoh, M.; Arifuku, K.; Sato, K.; Takui, T. Nat. Mater. 2011, 10, 947.

[22] Yang, D.-H.; Yao, Z.-Q.; Wu, D.; Zhang, Y.-H.; Zhou, Z.; Bu, X.-H. J. Mater. Chem. A 2016, 4, 18621

[23] Xu, F.; Jin, S.; Zhong, H.; Wu, D.; Yang, X.; Chen, X.; Wei, H.; Fu, R.; Jiang, D. Sci. Rep. 2015, 5, 8225.

[24] Wang, S.; Wang, Q.; Shao, P.; Han, Y.; Gao, X.; Ma, L.; Yuan, S.; Ma, X.; Zhou, J.; Feng, X.; Wang, B. J. Am. Chem. Soc. 2017, 139, 4258.

[25] Lei, Z.; Yang, Q.; Xu, Y.; Guo, S.; Sun, W.; Liu, H.; Lv, L. P.; Zhang, Y.; Wang, Y. Nat. Commun. 2018, 9, 576.

[26] (a) Bachman, J. C.; Muy, S.; Grimaud, A.; Chang, H. H.; Pour, N.; Lux, S. F.; Paschos, O.; Maglia, F.; Lupart, S.; Lamp, P.; Giordano, L.; Shao-Horn, Y. Chem. Rev. 2016, 116, 140. (b) Thangadurai, V.; Narayanan, S.; Pinzaru, D. Chem. Soc. Rev. 2014, 43, 4714.

[27] (a) Richards, W. D.; Miara, L. J.; Wang, Y.; Kim, J. C.; Ceder, G. Chem. Mater. 2015, 28, 266. (b) Xin, S.; You, Y.; Wang, S.; Gao, H.-C.; Yin, Y.-X.; Guo, Y.-G. ACS Energy Lett. 2017, 2, 1385. (c) Jeong, K.; Park, S.; Lee, S.-Y. J. Mater. Chem. A 2019, 7, 1917.

[28] (a) Zhang, H.; Li, C.; Piszcz, M.; Coya, E.; Rojo, T.; Rodriguez-Martinez, L. M.; Armand, M.; Zhou, Z. Chem. Soc. Rev. 2017, 46, 797. (b) Bouchet, R.; Maria, S.; Meziane, R.; Aboulaich, A.; Lienafa, L.; Bonnet, J.-P.; Phan, T. N. T.; Bertin, D.; Gigmes, D.; Devaux, D.; Denoyel, R.; Armand, M. Nat. Mater. 2013, 12, 452.

[29] Du, Y.; Yang, H.; Whiteley, J. M.; Wan, S.; Jin, Y.; Lee, S. H.; Zhang, W. Angew. Chem. Int. Ed. 2016, 55, 1737.

[30] Chen, H.; Tu, H.; Hu, C.; Liu, Y.; Dong, D.; Sun, Y.; Dai, Y.; Wang, S.; Qian, H.; Lin, Z.; Chen, L. J. Am. Chem. Soc. 2018, 140, 896.

[31] Guo, Z.; Zhang, Y.; Dong, Y.; Li, J.; Li, S.; Shao, P.; Feng, X.; Wang, B. J. Am. Chem. Soc. 2019, 141, 1923.

[32] Xu, Q.; Tao, S.; Jiang, Q.; Jiang, D. J. Am. Chem. Soc. 2018, 140, 7429.

[33] Zhang, G.; Hong, Y. L.; Nishiyama, Y.; Bai, S.; Kitagawa, S.; Hori- ke, S. J. Am. Chem. Soc. 2019, 141, 1227.

[34] (a) Liu, X.; Huang, J. Q.; Zhang, Q.; Mai, L. Adv. Mater. 2017, 29, 1601759. (b) Bruce, P. G.; Freunberger, S. A.; Hardwick, L. J.; Tarascon, J. M. Nat. Mater. 2011, 11, 19. (c) Pang, Q.; Liang, X.; Kwok, C. Y.; Nazar, L.F. Nat. Energy 2016, 1, 16132.

[35] (a) Yin, Y. X.; Xin, S.; Guo, Y. G.; Wan, L. J. Angew. Chem. Int. Ed. 2013, 52, 13186. (b) Ji, X.; Lee, K. T.; Nazar, L. F. Nat. Mater. 2009 8, 500. (c) Zhao, Y.; Wu, W.; Li, J.; Xu, Z.; Guan, L. Adv. Mater. 2014, 27, 1694. (d) Cheng, Z.; Pan, H.; Zhong, H.; Xiao, Z.; Li, X.; Wang, R. Adv. Funct. Mater. 2018, 28, 1707597.

[36] (a) Song, J.; Gordin, M. L.; Xu, T.; Chen, S.; Yu, Z.; Sohn, H.; Lu, J.; Ren, Y.; Duan, Y.; Wang, D. Angew. Chem. Int. Ed. 2015, 54 , 4325. (b) Yang, C. P.; Yin, Y. X.; Ye, H.; Jiang, K. C.; Zhang, J.; Guo, Y. G. ACS Appl. Mater. Interfaces 2014, 6, 8789.

[37] Liao, H.; Ding, H.; Li, B.; Ai, X.; Wang, C. J. Mater. Chem. A 2014, 2,8854 .

[38] Liao, H.; Wang, H.; Ding, H.; Meng, X.; Xu, H.; Wang, B.; Ai, X.; Wang, C. J. Mater. Chem. A 2016, 4, 7416.

[39] Meng, Y.; Lin, G.; Ding, H.; Liao, H.; Wang, C. J. Mater. Chem. A 2018, 6, 17186

[40] Xu, F.; Yang, S.; Jiang, G.; Ye, Q.; Wei, B.; Wang, H. ACS Appl. Mater. Interfaces 2017, 9, 37731.

[41] (a) Mclntosh, S.; Gorte, R. J. Chem. Rev. 2004, 104, 4845. (b) Winter, M.; Brodd, R. J. Chem. Rev. 2004, 104, 4245.

[42] (a) Schmidt-Rohr, K.; Chen, Q. Nat. Mater. 2008, 7, 75. (b) Mauritz, K. A. Chem. Rev. 2004, 104, 4535. (c) Kreuer, K.-D.; Paddison, S. J.; Spohr, E.; Schuster, M. Chem. Rev. 2004, 104, 4637.

[43] (a) Devanathan, R. Energy Environ. Sci. 2008, 1, 101. (b) Peckham, T. J.; Holdcroft, S. Adv. Mater. 2010, 22, 4667.

[44] (a) Rikukawa, M.; Sanui, K. Prog. Polym. Sci. 2000, 25, 1463. (b) Paddison, S. J. Annu. Rev. Mater. Res. 2003, 33, 289.

[45] (a) Horike, S.; Umeyama, D.; Kitagawa, S. Acc. Chem. Res. 2013 46, 2376. (b) Hurd, J. A.; Vaidhyanathan, R.; Thangadurai, V.; Ratcliffe, C. I.; Moudrakovski, I. L.; Shimizu, G. K. Nat. Chem. 2009, 1, 705. (c) Furukawa, H.; Cordova, K. E.; O'Keeffe, M.; Yaghi, O. M. Science 2013, 341, 1230444.

[46] Chandra, S.; Kundu, T.; Kandambeth, S.; Babarao, R.; Marathe, Y.; Kunjir, S. M.; Banerjee, R. J. Am. Chem. Soc. 2014, 136, 6570.

[47] Xu, H.; Tao, S.; Jiang, D. Nat. Chem. 2016, 15, 722.

[48] Chandra, S.; Kundu, T.; Dey, K.; Addicoat, M.; Heine, T.; Banerjee, R. Chem. Mater. 2016, $28,1489$.

[49] Sasmal, H. S.; Aiyappa, H. B.; Bhange, S. N.; Karak, S.; Halder, A.; Kurungot, S.; Banerjee, R. Angew. Chem. Int. Ed. 2018, 57, 108.

[50] Chen, X.; Paul, R.; Dai, L. Natl. Sci. Rev. 2017, 4, 453.

[51] Li, X.; Wei, B. Nano Energy 2013, 2, 159.

[52] Wang, Y.; Song, Y.; Xia, Y. Chem. Soc. Rev. 2016, 45, 5925.

[53] DeBlase, C. R.; Silberstein, K. E.; Truong, T. T.; Abruna, H. D.; Dichtel, W. R. J. Am. Chem. Soc. 2013, 135, 16821

[54] DeBlase, C. R.; Hernandez-Burgos, K.; Silberstein, K. E.; Rodrıguez-Calero, G. G.; Bisbey, R. P.; Abruña, H. D.; Dichtel, W. R. ACS Nano 2015, 9, 3178.

[55] Mulzer, C. R.; Shen, L; Bisbey, R. P.; McKone, J. R.; Zhang, N.; Abruña, H. D.; Dichtel, W. R. ACS Cent. Sci. 2016, $2,667$.

[56] Xu, F.; Xu, H.; Chen, X.; Wu, D.; Wu, Y.; Liu, H.; Gu, C.; Fu, R.; Jiang, D. Angew. Chem. Int. Ed. 2015, 54, 6814.

[57] Chandra, S.; Roy Chowdhury, D.; Addicoat, M.; Heine, T.; Paul, A.; Banerjee, R. Chem. Mater. 2017, 29, 2074.

[58] Stamenkovic, V. R.; Strmcnik, D.; Lopes, P. P.; Markovic, N. M. Nat. Mater. 2016, 16, 57.

[59] Stamenkovic, V. R.; Strmcnik, D.; Lopes, P. P.; Markovic, N. M. Nat. Mater. 2016, 16, 57.

[60] Fujishima, A.; Honda, K. Nature 1972, 238, 37.

[61] (a) Kudo, A.; Miseki, Y. Chem. Soc. Rev. 2009, 38, 253. (b) Chen, S.; Takata, T.; Domen, K. Nat. Rev. Mater. 2017, 2, 17050.

[62] (a) Wang, X.; Maeda, K.; Thomas, A.; Takanabe, K.; Xin, G.; Carlsson, J. M.; Domen, K.; Antonietti, M. Nat. Mater. 2009, 8, 76. (b) Schwinghammer, K.; Mesch, M. B.; Duppel, V.; Ziegler, C.; Senker, J.; Lotsch, B. V. J. Am. Chem. Soc. 2014, 136, 1730.

[63] (a) Sprick, R. S.; Jiang, J. X.; Bonillo, B.; Ren, S.; Ratvijitvech, T.; Guiglion, P.; Zwijnenburg, M. A.; Adams, D. J.; Cooper, A. I. J. Am. Chem. Soc. 2015, 137, 3265. (b) Li, L.; Cai, Z.; Wu, Q.; Lo, W. Y.; Zhang, N.; Chen, L. X.; Yu, L. J. Am. Chem. Soc. 2016, 138, 7681 (c) Yang, C.; Ma, B. C.; Zhang, L.; Lin, S.; Ghasimi, S.; Landfester K.; Zhang, K. A.; Wang, X. Angew. Chem. Int. Ed. 2016, 55, 9202.

[64] (a) Woods, D. J.; Sprick, R. S.; Smith, C. L.; Cowan, A. J.; Cooper, 
A. I. Adv. Energy Mater. 2017, 7, 1700479. (b) Sprick, R. S.; Bonillo, B.; Clowes, R.; Guiglion, P.; Brownbill, N. J.; Slater, B. J.; Blanc, F.; Zwijnenburg, M. A.; Adams, D. J.; Cooper, A. I. Angew. Chem. Int. Ed. 2016, 55, 1792.

[65] Stegbauer, L.; Schwinghammer, K.; Lotsch, B. V. Chem. Sci. 2014, 5,2789 .

[66] Vyas, V. S.; Haase, F.; Stegbauer, L.; Savasci, G.; Podjaski, F.; Ochsenfeld, C.; Lotsch, B. V. Nat. Commun. 2015, 6, 8508.

[67] Pachfule, P.; Acharjya, A.; Roeser, J.; Langenhahn, T.; Schwarze, M.; Schomacker, R.; Thomas, A.; Schmidt, J. J. Am. Chem. Soc. 2018, 140, 1423 .

[68] Wang, X.; Chen, L.; Chong, S. Y.; Little, M. A.; Wu, Y.; Zhu, W. H.; Clowes, R.; Yan, Y.; Zwijnenburg, M. A.; Sprick, R. S.; Cooper, A. I. Nat. Chem. 2018, 10, 1180.

[69] Berardi, S.; Drouet, S.; Francàs, L.; Gimbert-Suriñach, C.; Guttentag, M.; Richmond, C.; Stoll, T.; Llobet, A. Chem. Soc. Rev. 2014, 43, 7501.

[70] (a) Reier, T.; Oezaslan, M.; Strasser, P. ACS Catal. 2012, 2, 1765. (b) Sardar, K.; Petrucco, E.; Hiley, C. I.; Sharman, J. D.; Wells, P. P.; Russell, A. E.; Kashtiban, R. J.; Sloan, J.; Walton, R. I. Angew. Chem. Int. Ed. 2014, 53, 10960.

[71] (a) Chang, J.; Xiao, Y.; Xiao, M.; Ge, J.; Liu, C.; Xing, W. ACS Catal. 2015, 5, 6874. (b) Zhang, C.; Antonietti, M.; Fellinger, T.-P. Adv. Funct. Mater. 2014, 24, 7655. (c) Wu, L.; Li, Q.; Wu, C. H.; Zhu, H.; Mendoza-Garcia, A.; Shen, B.; Guo, J.; Sun, S. J. Am. Chem. Soc. 2015, 137, 7071. (d) Zhang, G.; Huang, C.; Wang, X. Small, 2015, $11,1215$.

[72] Blakemore, J. D.; Crabtree, R. H.; Brudvig, G. W. Chem. Rev. 2015, $115,12974$.

[73] Aiyappa, H. B.; Thote, J.; Shinde, D. B.; Banerjee, R.; Kurungot, S. Chem. Mater. 2016, $28,4375$.

[74] Mullangi, D.; Dhavale, V.; Shalini, S.; Nandi, S.; Collins, S.; Woo,
T.; Kurungot, S.; Vaidhyanathan, R. Adv. Energy Mater. 2016, 6, 1600110.

[75] Nandi, S.; Singh, S. K.; Mullangi, D.; Illathvalappil, R.; George, L.; Vinod, C. P.; Kurungot, S.; Vaidhyanathan, R. Adv. Energy Mater. 2016, 6, 1601189

[76] (a) Lewis, N. S.; Nocera, D. G. Proc. Natl. Acad. Sci. U. S. A. 2006, 103, 15729. (b) Gray, H. B. Nat. Chem. 2009, 1, 7 .

[77] (a) Zhao, G.; Huang, X.; Wang, X.; Wang, X. J. Mater. Chem. A 2017, 5, 21625. (b) Habisreutinger, S. N.; Schmidt-Mende, L.; Stolarczyk, J. K. Angew. Chem. Int. Ed. 2013, 52, 7372. (c) Inoue, T.; Fujishima, A.; Konishi, S.; Honda, K. Nature 1979, 277, 637. (d) Thampi, K. R.; Kiwi, J.; Grätzel, M. Nature 1987, 327, 506. (e) Tu, W.; Zhou, Y.; Zou, Z. Adv. Mater. 2014, 26, 4607.

[78] (a) Lin, W.; Frei, H. J. Am. Chem. Soc. 2005, 127, 1610. (b) Anpo, M.; Takeuchi, M. J. Catal. 2003, 216, 505. (c) Shioya, Y.; Ikeue, K.; Ogawa, M.; Anpo, M. Appl. Catal. A: General 2003, 254, 251. (d) Matsuoka, M.; Anpo, M. J. Photochem. Photobiol. C: Photochem. Rev. 2003, 3, 225. (e) Anpo, M.; Yamashita, H.; Ikeue, K.; Fujii, Y.; Zhang, S. G.; Ichihashi, Y.; Park, D. R.; Suzuki, Y.; Koyano, K.; Tatsumi, T. Catal. Today 1998, 44, 327. (f) Anpo, M. J. $\mathrm{CO}_{2}$ Util. 2013, 1, 8. (g) Schneider, J.; Matsuoka, M.; Takeuchi, M.; Zhang, J.; Horiuchi, Y.; Anpo, M.; Bahnemann, D. W. Chem. Rev. 2014, 114, 9919.

[79] Yang, S.; Hu, W.; Zhang, X.; He, P.; Pattengale, B.; Liu, C.; Cendejas, M.; Hermans, I.; Zhang, X.; Zhang, J.; Huang, J. J. Am. Chem. Soc. 2018, 140, 14614.

[80] Lin, S.; Diercks, C. S.; Zhang, Y.-B.; Kornienko, N.; Nichols, E. M.; Zhao, Y.; Paris, A. R.; Kim, D.; Yang, P.; Yaghi, O. M.; Chang, C. J. Science 2015, 349, 1208.

[81] Diercks, C. S.; Lin, S.; Kornienko, N.; Kapustin, E. A.; Nichols, E. M.; Zhu, C.; Zhao, Y.; Chang, C. J.; Yaghi, O. M. J. Am. Chem. Soc. 2018, $140,1116$.

(Cheng, B.) 